\title{
Understanding bikeability: a methodology to assess urban networks
}

\author{
Giulia Reggiani ${ }^{1}$ (D) Tim van Oijen ${ }^{1} \cdot$ Homayoun Hamedmoghadam ${ }^{2}$. \\ Winnie Daamen ${ }^{1} \cdot$ Hai L. Vu$^{2}$. Serge Hoogendoorn ${ }^{1}$
}

Accepted: 24 May 2021 / Published online: 7 June 2021

(c) The Author(s) 2021

\begin{abstract}
A fully separated bicycle network from vehicular traffic is not realistic even for the most bicycle-friendly cities. Thus, all around the world urban cycling entails switching between streets of different safety, convenience, and comfort levels. As a consequence, the quality of bicycle networks should be evaluated not based on one but multiple factors and by considering the different user preferences regarding these factors. More comprehensive methodologies to assess urban bicycle networks are essential to the operation and planning of modern city transportation. This work proposes a multi-objective methodology to assesswhat we refer to as-bikeability between origin-destination locations and over the entire network, useful for evaluation and planning of bicycle networks. We do so by introducing the concept of bikeability curves which allows us to assess the quality of cycling in a city network with respect to the heterogeneity of user preferences. The application of the proposed methodology is demonstrated on two cities with different bike cultures: Amsterdam and Melbourne. Our results suggest the effectiveness of bikeability curves in describing the characteristic features and differences in the two networks.
\end{abstract}

Keywords Bikeability - Complex network analysis - Urban traffic networks · Multiobjective optimisation $\cdot$ Multi-layer networks

\section{Introduction}

Few cities have a fully connected network of separated bike tracks. Often, bike networks consist of a heterogeneous set of streets (including car streets) with various comfort and convenience levels. This forces cyclists to switch between networks with different comfort levels during their journey (as if they travel through a multi-layer network). Unlike drivers of private vehicles, cyclists choose their routes by taking multiple contrasting objectives

Giulia Reggiani

g.reggiani@tudelft.nl

1 Transport and Planning Department, Faculty of Civil Engineering and Geosciences (CiTG), TU Delft, Stevinweg 1, 2628 CN Delft, The Netherlands

2 Institute of Transport Studies, Faculty of Engineering, Monash University, Melbourne, Australia 
into consideration, for example, they consider the distance and suitability of a bike route (Ehrgott et al. 2012) while also accounting for safety and comfort (Stinson and Bhat 2003).

Cyclists have heterogeneous route preferences and therefore, one single route that is optimally bikeable for all individuals does generally not exist between an origin-destination (OD). For example, commuter cyclists might prefer a very short bike route with high discomfort while recreational cyclists might prefer a longer route that is more comfortable (Krizek et al. 2007). Many studies have successfully modeled cyclists' route choice behaviour and have reported findings concerning the average cyclists' route preference in terms of detour, facility type, travel time, etc. (Krizek et al. 2007; Menghini et al. 2010; Broach et al. 2012; Zimmermann et al. 2017). While the average behaviour is useful for understanding attractors and deterrents of cyclists in general, it does not provide a comprehensive picture of individual facets (since averagin blurs out the effects of diversity among cyclists). We think that network assessment tools should provide a full picture of what the network supplies means to all users, not only to the average cyclist. Since transparency and understanding of the network quality are key elements to decision makers we aim to provide a tool to evaluate the network free from user preference assumptions. It is then up to the policymaker to slice the analysis for a specific user type she wants to serve.

The heterogeneity of both the street network and of users' preferences motivates the development of a multi-objective methodology to assess the bikeability of a network. Having multiple objectives offers a more comprehensive evaluation (in terms of user heterogeneity) of the network, but also makes the evaluation more complex (Gholamialm and Matisziw 2018). This work addresses this problem via a network-wide bikeability curve concept we elaborate on. The main contribution is to define an assumption-free methodology for assessing how bikeable specific OD pairs and the networks themselves are, by taking into account the heterogeneity of the streets and users' preferences. To this end, this work provides a multi-objective tool for city planners to study bikeability of specific OD pairs, neighbourhoods or a whole city network.

This article is organized as follows. "Literature review" section presents a literature review on bikeability assessment methodologies. "Multi-layered bikeability assessment methodology" section illustrates the proposed methodology. Implementation of a key component of our methodology (the discomfort function) is discussed in "Implementation of the discomfort function" section. An application of the methodology is presented in "Case study: Amsterdam-Melbourne" section via case-studies of Amsterdam, Netherlands and Melbourne, Australia. After discussing the applications of the proposed framework and implications for future research in "Discussion" section, the conclusions are presented in "Conclusions" section.

\section{Literature review}

Answering the question "how easily can a person get to their destination by riding a bike?" is a non-trivial task in complex urban networks, while it is crucial for measuring the goodness of a bike network. In some previous studies, this question has been mostly answered by focusing on network structural properties and neglecting user preferences. For example, Dill (2004) explores a wide range of connectivity measures from various domains such as landscape ecology, geography, and urban planning. Whereas Schoner and Levinson (2014) and Orozco et al. (2020) use network science to measure connectivity via indicators such 
as network completeness and the number of connected components. However, the comfort and safety of a bicycle network is a crucial component for its assessment, as it reveals to be an important factor affecting cyclists' route choices (Stinson 1828). For this reason, to assess urban bicycle networks, we refer to the concept of "bikeability".

Bikeability can be defined as the extent to which an environment is convenient and safe for cycling. However, a recent review of the existing literature shows that bikeability has been defined and assessed in several ways and the study concludes that there is no universally accepted definition (Kellstedt et al. 2021). Bikeability is the combination of objective and subjective factors and it integrates concepts such as bicycle comfort, bicycle suitability, bicycle friendliness, and bicycle accessibility. According to a review published in 2020 (Arellana et al. 2020) comfort and safety were the most common factors to construct bikeability indexes, around $96 \%$ of the reviewed studies included at least one of these factors. Lowry et al. (2012) present bikeability in relation to the scale of analysis and distinguish between suitability of a link and bikeability defined as the convenience and comfort of routes over the entire city network. In our work, bikeability focuses on the detour and type (or presence) of physical bicycle infrastructure, which can be regarded both as a comfort and a safety factor (Arellana et al. 2020). The scale of the assessment developed in this study is based on segment level measurements of length and comfort at a city- or neighbourhood-level analysis.

The concept of comfort introduces user preferences and the trade-off between directness versus comfort to the analysis. Cyclists have different route preferences: some may prefer direct routes, while others prefer more comfortable ones. Previous studies have made it clear that cyclists are willing to choose a longer route over the shortest path in order to ride bicycle-designated streets, i.e., more comfortable and safer routes (Sener et al. 2009; Larsen and El-Geneidy 2011; Broach et al. 2012; Hood et al. 2011; Krizek et al. 2007). Detours depend on trip length (Larsen and El-Geneidy 2011; Krizek et al. 2007) and cyclists who cycle regularly are less likely to use a bicycle facility (Larsen and El-Geneidy 2011). On average recreational and afternoon cyclists tend to have larger detours compared to morning commuters (Krizek et al. 2007; Hyodo et al. 2000) thanks to fewer time constraints that allow longer travel times.

The average detour rates of trips range between $8 \%$ and 93\%. In Broach et al. (2012), Winters et al. (2010) and Boisjoly et al. (2020), 50-75\% of observed trips deviate from the shortest route by $10 \%$. Hood et al. (2011) analyse marginal rates of substitution and show that the average cyclist is willing to add a mile on bike lanes in exchange for only half a mile on ordinary roads. Evidence from Minneapolis states that on average cyclists travelled $67 \%$ longer in order to include a cycle path facility on their route whereas weekend cyclists are willing to cycle up to $40 \%$ more (resulting in 93\% detour) (Krizek et al. 2007). Figure 1 manifests the variety amongst different cyclists and contexts when it comes to the willingness to make detours, which supports our motivation to define an assumption-free assessment tool.

A well-known study that has incorporated the interplay between comfort and distance is Mekuria et al. (2012). Mekuria et al. (2012) and studies following a similar approach (Furth et al. 2016; Lowry and Loh 2017; Abad and van der Meer 2018) define the preference of the users as an a priori specification of tolerance level of traffic stress. To analyse low-stress connectivity, these studies assume that a trip is connected at a certain level of stress if all segments of the trip are below that stress level and if the detour is below a fixed rate. These are a priori assumptions that may limit the analysis on specific user types. For example, one may be willing to accept a high-stress level for a small part of the trip to avoid large detours. Moreover, the accepted detour ratio depends on the user (different users may accept different 


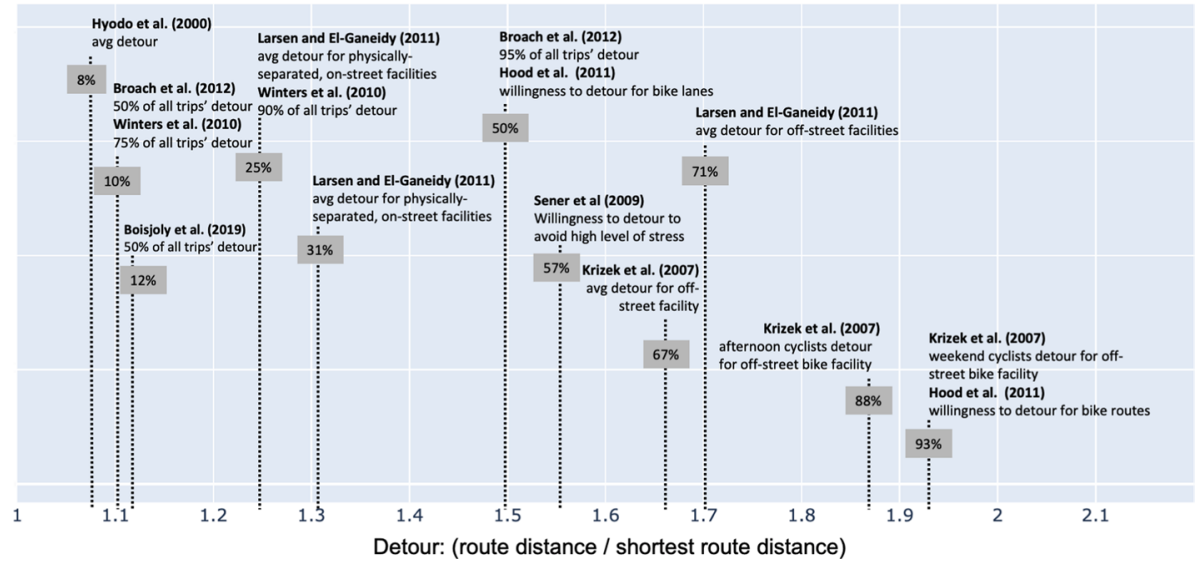

Fig. 1 Overview of cyclists' detour behaviour and bike facility preference reported in the literature

detour ratios) and on the gained comfort. We believe that it is useful to observe how the network performs when assumptions on detour and comfort are not fixed, as fixing preferences a priori may obscure the complete picture of the network bikeability and results in an analysis that is highly dependent on the assumption parameters. In order to not make assumptions on the preferred comfort level of users, our work will not reduce bikeability to one aggregated value. Instead, we represent it as a set of bikeability curves that can be aggregated to analyse the quality of the entire city network. This allows us to analyse the network depending on all possible user preferences without the need to specify their behaviour beforehand.

Instead of using a generalised cost function, which requires a priori assumptions on users, we model route choice as a multi-objective behaviour. This allows us to also assess the network in an assumption-free method. Although a few existing studies (Ehrgott et al. 2012; Kang and Fricker 2018; Wang et al. 2018) has used multi-objective criteria in route choice modelling no previous study, except (Gholamialm and Matisziw 2018), has attempted to assess a bicycle network with it. The work of Gholamialm and Matisziw (2018) points out the higher complexity of analysing optimal route alternatives for multiple ODs compared to only one OD and addresses it with aggregated summary metrics which only reflect a partial picture of bikeability. Summary matrices are computed for one criterion at a time, meaning that they do not show the trade-off between the different criteria [see the method section in Gholamialm and Matisziw (2018)].

Our methodology extends the multi-objective bikeability assessment literature by providing a more general framework that avoids summary metrics, to observe bikeability depending on all the different user preferences. We tackle the increased complexity of multi-objective analysis by providing a methodology to build an average bikeability curve: an intuitive and visual instrument to evaluate a network. This work develops a methodology for the analysis and visualisation of bikeability that uses the shape of Pareto fronts (Khorram et al. 2014) to extract information. We validate and illustrate the use of our proposed method by assessing and visualising the network-wide bikeability of two cities. 


\section{Multi-layered bikeability assessment methodology}

We do not assume that the users will-or in fact, can-always cycle on streets that do not exceed their tolerance of discomfort. For example, one could prefer separated cycle tracks (that have a low level of discomfort) but be willing to take a higher risk or discomfort and cycle along with vehicular traffic for a short part of the trip, if this avoids a very long detour. This is also because only separated cycle tracks will not—at least for many cities-make a pathway from an origin to the desired destination. Thus cyclists go through a multi-objective optimisation process to select their route comprised of cycling tracks and other types of roads.

In general, multiple objectives should be considered simultaneously for assessing a bicycle network. To name just a few we can consider: traveled distance, perceived safety, number of interruptions, and comfort. For a matter of simplicity, we illustrate our methodology for two representative costs: distance and discomfort. Distance is an objective factor whereas discomfort is a subjective, and complex factor to measure (Arellana et al. 2020). The methodology still holds for a wider set of objectives at the cost of less interpretability. ${ }^{1}$ In the following paragraphs, we illustrate how the problem of assessing bikeability between a pair of nodes can be seen as a trade-off, between distance and discomfort, on a multi-layer network.

We claim that there is no universally accepted definition of what a bike network is. As Mekuria et al. (2012) points out, there are two perspectives on bicycle networks: from a municipality point of view, a bike network is made up of any street where cycling is not prohibited, whereas, from a user perspective a bike network is a set of streets and paths that do not exceed the person's tolerance for traffic stress. We do not narrow down a bike network to only the separated bike streets where all users feel comfortable to cycle on. Instead, we include all the different streets, from most to least comfortable ones, by defining the bike network as a multi-layer network (see "Modelling the multi-layer network" section for the formal definition) where each layer represents a sub-network made of all roads with homogeneous discomfort. In a multi-layer representation (Boccaletti et al. 2014; Kivelä et al. 2014) of a bike network, a route becomes a set of links on a set of layers in addition to a set of links connecting the different layers. Figure 2 provides a visual representation of a route as a path on a multi-layered graph. A multi-layer network effectively shows the costs a cyclist incurs when cycling from an origin to a destination. This model allows us to generalize the discomfort cost of a route depending on the comfort level of the street segments that define the route and the number of changes between comfort levels. Thus, the discomfort of a trip can be defined as the sum of the discomfort of all the links and nodes in the path.

Hereafter, we first define what is meant by a multi-layer network ("Modelling the multilayer network" section), secondly we present route choice as an optimisation problem based on the concept of disutility (in "Individual route choice model to define bikeability" section ). Subsequently, the set of solutions to the route choice problem is identified by the Pareto front (in "Pareto optimality" section). Using a modified Dijkstra algorithm the Pareto optimal (set of) bike routes that connect an OD pair are identified ("Multi-objective shortest path algorithm" section). Finally, we define the bikeability curves for one OD pair

\footnotetext{
1 For insight about additional complexity of multi-objective optimisation as more objectives are added we refer the reader to Jakob and Blume (2014).
} 
Fig. 2 Example of a route from node 1 to node 6 using intra-layer links on different layers (solid links) and switching between layers represented by inter-layer links (dashed arrows)

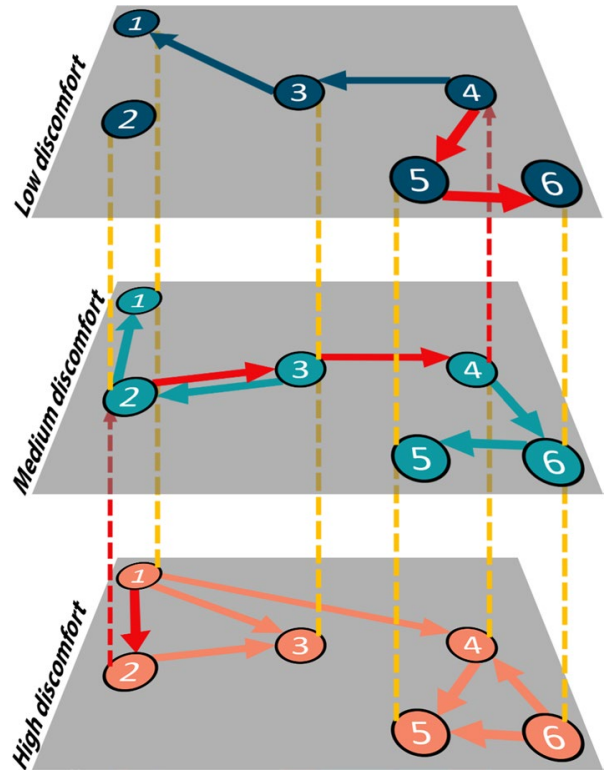

and for a network (in "Definition of the bikeability curve" and "Network-wide bikeability curve" sections).

\section{Modelling the multi-layer network}

The urban bicycle road network is modelled as a multi-layered directed network denoted by $G=(N, E, L)$, where $N$ is the set of all nodes, $E$ is the set of directed links and $L$ is a set of layers. Each intersection $i$ between the edges is represented by a single node $n_{i}^{\ell} \in N$, in each layer $\ell \in L$ of the network. Each link $e \in E$ can be denoted as an ordered pair $e=\left(n_{i}^{\ell}, n_{j}^{\ell^{\prime}}\right)$ representing a connection from node $i$ in layer $\ell$ to node $j$ in layer $\ell^{\prime}$. Intra-layer links $\left(n_{i}^{\ell}, n_{j}^{\ell}\right) \in E$ (i.e. connecting two nodes on the same layer) represent the real connections between different locations by streets of the same facility type. Inter-layer links only connect the corresponding nodes between different layers, i.e. $\forall e=\left(n_{i}^{\ell}, n_{j}^{\ell^{\prime}}\right) \ell \neq \ell^{\prime} \Rightarrow i=j$. These links are used to model the change of facility type while cycling. In order to address the source node of a link, we introduce $\mathcal{S}: E \rightarrow N$ as the function that maps a link onto its source node. To address the layer of a link, we introduce $\mathcal{L}: E \rightarrow L$ as the function that maps a link onto the layer of its source node. Each link has a length and discomfort attribute which differs across different network layers. The length of an intra-layer link is the physical length of the street segment, whereas the length of inter-layer links represents the length of the "turning manoeuvre", and can be a few tens of meters, for a wide intersection, or set to a small but positive value, for an immediate transition. ${ }^{2}$ The discomfort attribute of an intra-layer link models the impedance of cycling on the particular link, which is highly influenced by its facility type, whereas the discomfort of inter-layer link models the inconvenience of changing cycling facility type. Empirical data have shown that such a

\footnotetext{
${ }^{2}$ Length should always be strictly positive for technical reasons (to prevent infinite looping of the path search algorithm).
} 
facility discontinuity implies (1) more diverse motion strategies of users, (2) speed reduction and (3) more maneuvers and braking compared to the control site (Niaki et al. 2018).

We define a directed path through the multi-layered network from node $o$ to node $d$ as an ordered sequence of links $\pi=\left(e_{1}, \ldots, e_{|\pi|}\right)$, with length $|\pi|$, where $e_{1}$ 's source and $e_{|\pi|}$ 's target are nodes $o$ and $d$ respectively. The total length of the path $\pi$ is calculated as

$$
D(\pi)=\sum_{e \in \pi} d(e)
$$

where $d(e)$ is the length of the edge $e$. Similarly, the total discomfort of path $\pi$ is calculated as a sum of path segment discomfort $r(e)$ :

$$
R(\pi)=\sum_{e \in \pi} r(e)
$$

The segment discomfort $r(e)$ is the combination of edge and node discomfort:

$$
r(e)=r_{e}(e)+r_{n}(\mathcal{S}(e)),
$$

where $r_{e}(e)$ is a discomfort function that maps an edge $e$ to a discomfort value, and $r_{n}(n)$ is a discomfort function that maps a node $n$ to a discomfort value, which can, for instance, be used to model negative impact on cyclists route choice [due to deceleration, stop, waiting time to traverse an intersection (Ton et al. 2017)]. We model $r_{n}(\mathcal{S}(e))$ to be always zero for the first edge that a person travels.

The methodology presented here is independent of the discomfort function. The only condition is that the discomfort should not be negative, since this could introduce cycles with a positive accumulated utility, which is not supported by our later introduced methodologies. Some examples of discomfort function specifications are reported in "Implementation of the discomfort function" section, where we discuss viable implementations.

\section{Individual route choice model to define bikeability}

In this section, we briefly touch upon route choice and utility theory to describe the individual route choice behaviour because bikeability should assess what the network offers, in relation to what the cyclist would choose. Thus, understanding how cyclists choose their routes guides us in defining the bikeability of a network.

Route choice modelers often represent user preferences via so-called (dis-) utility functions (Ben-Akiva et al. 2004). Each route has specific qualities or costs that result in a utility value perceived by the cyclist. Cyclists are assumed to be rational users, thus to be utility maximisers (or disutility minimisers). Without loss of generality, in this work we simplify route choice by assuming that route choice depends only on two major factors such as distance and discomfort ${ }^{3}$ factor. The rational cyclist will typically identify the most bikeable route as the one with a distance-discomfort combination associated with the lowest disutility, as defined in (1).

\footnotetext{
${ }^{3}$ Discomfort depends on many factors (both observable and non-observable) (Arellana et al. 2020), here we model discomfort as an ideal impedance value that one can measure to the best of its data availability. Later in the case study we will measure discomfort as presence and type of bicycle infrastructure, which is regarded by some studies as a comfort and others as a safety factor (Arellana et al. 2020).
} 
Fig. 3 Solution to the route choice optimisation problem for one OD pair, in the case of linear cost function $C(D(\pi), R(\pi))=\beta * D(\pi)+\gamma * R(\pi)$
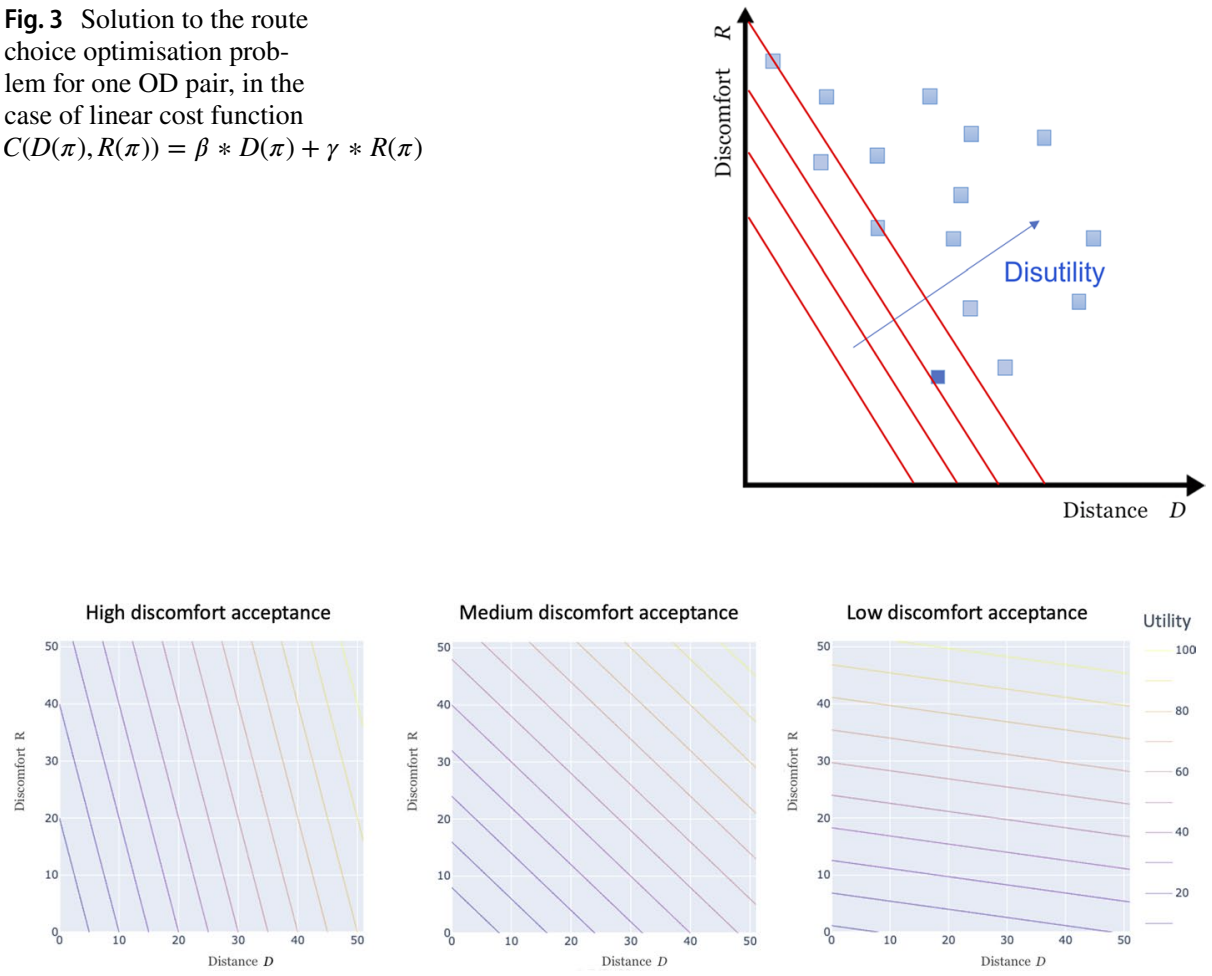

Fig. 4 Indifference curves reflecting discomfort acceptance attitude of cyclists

The route choice problem can be seen as an optimisation problem where each individual will minimise its total disutility constrained to the set of feasible routes. Let us define $\Pi$ as the set of all feasible routes from $o$ to $d$, and let us define $C(D(\pi), R(\pi)): \mathbb{R} \times \mathbb{R} \rightarrow \mathbb{R}$ as the cost function that maps a pair of total distance and total discomfort values to a cost value. Now, the individual route choice problem can be formally defined as:

$$
\pi^{*}=\operatorname{argmin}_{\pi \in \Pi} C(D(\pi), R(\pi)) .
$$

The solution to the optimisation problem of Eq. (1) is depicted in Fig. 3 where we plot the iso-disutility lines (level sets of $C(D(\pi), R(\pi)$ )) and the feasible set of routes $\Pi$ (as points) with respect to a linear utility function. Graphically, the optimal (most bikeable) route corresponds to the route laying on the iso-disutility line closest to the origin. Users might also choose non-optimal routes, we leave the investigation of indifference bands (Vreeswijk et al. 2013) in the field of cycling to future research (see the discussion in "Discussion" section).

The shape of the iso-utility curves depends on individual preference and is generally not restricted to linear ones. Even if we would expect a linear cost function, the specific slopes of the indifference curves will differ between individuals. Figure 4, for example, shows different types of indifference curves reflecting the discomfort acceptance behaviour of cyclists. Depending on the indifference curve being used, the solution to the optimisation problem formulated by Eq. (1) is different, and consequently also the most bikeable route. 
In order to consider the entire set of individual optima, the following section introduces Pareto optimality.

\section{Pareto optimality}

In the previous section, we described how the chosen route of a cyclist is assumed to be the solution to an individual optimisation problem, in which the cyclist makes a trade-off between the experienced discomfort and travel distance. The extent to which individuals prefer short trips over comfortable trips defines the shape of their indifference curves and herewith the route that they will select. As a result, one single route that is optimal to all individuals generally does not exist, which makes the definition of a bikeability measure in this context far from trivial. Since a bikeability measure should consider the different users of the system, the measure should somehow incorporate the entire set of individual optimal routes.

Although we cannot identify a global optimal route, we can simplify our analysis by making the assumption that individuals will never prefer a certain route over another one if this route is both longer and more uncomfortable (non-dominant alternatives). Formally, this assumption can be called the "rational cyclist" assumption and can be expressed as:

$$
\frac{\partial C(d, r)}{\partial d}>0 \text { and } \frac{\partial C(d, r)}{\partial r}>0
$$

This assumption allows us to identify the smallest sub-set of routes which is guaranteed to contain each possible individual-specific optimal route (according to (1)). This sub-set is called the Pareto-front and deals with the concept of Pareto optimality. Pareto optimality of a multi-objective optimisation problem is defined as a situation in which it is impossible to score better on one criterion without scoring worse on another criterion. The set of all Pareto optimal choices is called the Pareto front. In the next sub-sections, we briefly discuss Pareto optimality and how this is applied to the route choice problem to identify the set of optimal routes.

\section{Pareto optimality for route choice problem}

In the context of the bicycle route choice problem on multi-layer networks, the concept of Pareto optimality is intertwined with the assumption that people will never prefer a route with a higher distance and discomfort (2).

Pareto domination of path $\pi^{1} \in \Pi$ over path $\pi^{2} \in \Pi$ is now defined as:

$$
\pi^{1} \leq_{P} \pi^{2} \Longleftrightarrow\left\{\begin{array}{l}
R\left(\pi^{1}\right) \leq R\left(\pi^{2}\right), \\
D\left(\pi^{1}\right) \leq D\left(\pi^{2}\right), \\
R\left(\pi^{1}\right)<R\left(\pi^{2}\right) \text { or } D\left(\pi^{1}\right)<D\left(\pi^{2}\right)
\end{array},\right.
$$

and the Pareto front $\Pi^{*}$ is defined as:

$$
\Pi^{*}=\left\{\pi^{1} \in \Pi \mid \nexists \pi^{2} \in \Pi, \pi^{2} \leq_{P} \pi^{1}\right\} .
$$

As mentioned before, with assumption (2), the optimal route according to (1), is guaranteed to be in $\Pi^{*}$ for each individual. This makes the assessment of the Pareto front a powerful methodology to study the bikeability of a network in terms of distance and discomfort. 

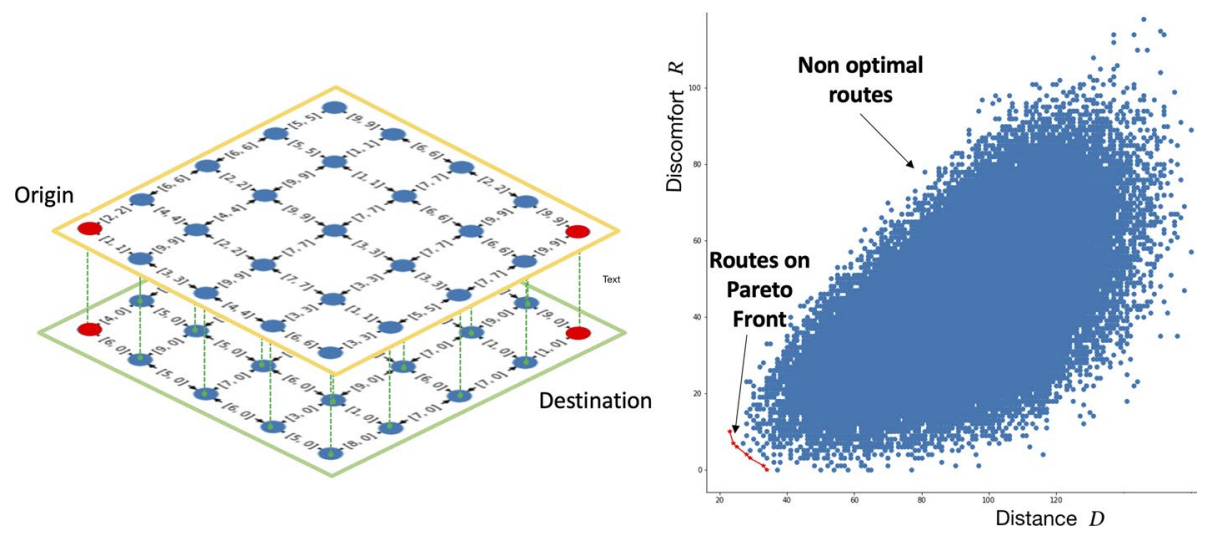

Fig. 5 Pareto front on $5 \times 5$ 2-layer grid network

We illustrate the aforementioned concepts in Fig. 5. As an example, we use a $5 \times 5$ 2-layer grid network. The two layers represent a very comfortable (green bordered layer) and uncomfortable (orange bordered layer) network. The intra-layer link weights (shown as ordered pairs of values), describe the length and discomfort of the links on the specific layer. The orange layer has a discomfort equals to the length of each link, whereas the green layer has a discomfort value of zero on each link. Thus, the total cost of moving on the orange layer is double the distance, whereas on the green layer the cost is equal to the distance. The only inter-layer links are between the same nodes, and, in this case, have zero length and zero discomfort value.

Finding all possible paths is a hard problem since there is an exponential number of simple paths (with respect to the size of the longest path). The scatter plot on the right shows 100,000 possible routes from the origin to the destination node. The routes were randomly generated excluding cycles (repeated nodes). Each route is a point on the distance-discomfort plane. Among the points, we color-coded the points on the Pareto front in red and joined them with a line. Note that in the example in Fig. 5 only 7 routes, among the millions of possible routes, are optimal with respect to distance and discomfort.

The shape of the Pareto front, also called "bikeability curve" in the remainder of this paper, will be used as the main tool to assess the bikeability in the multi-layered network. The Pareto front is identified by a modified version of the Dijkstra algorithm presented in "Multi-objective shortest path algorithm" section.

\section{Multi-objective shortest path algorithm}

Finding the shortest path on a network for a single objective is a well-known problem, solved in an exact way using Dijkstra's algorithm. In the presented methodology a multiobjective shortest path (MOSP) algorithm is needed since we want to minimise conflicting objectives of detour and discomfort. In literature, there is a wide variety of MOSP algorithms that work on the k-objectives optimal path problem. Algorithms can be classified as an exact, heuristic, approximate and meta-heuristic. In the field of transport, the 2-D problem was already addressed in 1979 in Dial (1979). The author describes an exact recursive method to find the Pareto front of a mode choice model in which cost and time are simultaneously being minimised. Another branch of exact methods to solve the MOSP problem is 
the family of labelling algorithms, in which lists of multi-dimensional labels are assigned to nodes, representing the set of non-dominated path costs (Hansen 1980; Martins 1984).

For our study, we implement a labelling algorithm in Python. The algorithm calculates the Pareto front for a given origin to all possible destination nodes. Since we applied our methodology only on small to medium-sized networks, we did not have to consider using more complex methods. For larger networks, the use of MOSP labelling algorithms can become infeasible due to the computation cost. For these cases, some exact methods have been developed, yielding a higher computational efficiency (e.g. Raith 2010; Duque et al. 2014). Also, an approximate method could be adopted, in which approximately non-dominated paths are found, for more insight we refer the reader to Garroppo et al. (2010). Given that the methodology presented can be generalized to more than two dimensions, for an extension of the multi-objective shortest path algorithm we refer the reader to Ghariblou et al. (2017) and Garroppo et al. (2010).

\section{Definition of the bikeability curve}

The bikeability curve, as presented in "Pareto optimality" section, is the Pareto front of routes which minimise two costs imposed on cyclists when choosing a route, in our case those are route distance and route discomfort. For the sake of comparison between routes, we normalise distances and discomfort values as explained in the following paragraphs. This enables us to use this methodology on OD pairs with different distances and to compare the discomfort values across routes.

The relative distance, also referred to as circuity in the remainder of the article, is the route distance (sum of all path links) divided by the Euclidean distance. This measure, used also in Schoner and Levinson (2014), allows identifying if there are OD pairs connected by meandering routes, which may suggest the need for new road infrastructure. Note that using Euclidean distance as a denominator can penalise cities whose topography imposes inevitable detours (e.g. cities with canals and bridges) and cities with many short ODs, since the effect of a detour is less for long routes than for short routes. The aforementioned limitations should be considered when comparing different cities. The purpose of this measure is to "identify recreationally oriented routes that meander or circle back on themselves, versus routes that provide an efficient utilitarian connection for commuting" as pointed out in Schoner and Levinson (2014).

The relative discomfort is defined as the total discomfort of the route (sum of discomfort on all links of the route) divided by the Euclidean distance (in meters) of the shortest route. The resulting relative discomfort represents the discomfort per meter. This allows for a comparison between discomfort values among the alternative routes.

We use Fig. 6a to explain the bikeability curve on the distance-discomfort space. Point $(A)$, in Fig. 6a, represents the shortest route. The ordinate of route $(A)$ shows the highest imposed discomfort on cyclists that seek to cycle the shortest route possible. Point $(B)$ is the shortest route. Its relative discomfort value shows the lowest possible discomfort level to go from origin to destination. Whereas $B$ 's relative distance value measures what is the imposed detour to have the least possible discomfort. Finally, an important measure on the curve is the trade-off rate (ToR) between any two routes on the Pareto front which is defined as $T o R=\Delta$ discomfort $/ \Delta$ distance between two routes. Ideally, the higher the tradeoff rate, the more bikeable the network structure as it means it is easier to go on a more comfortable route with a small increase in path length. 


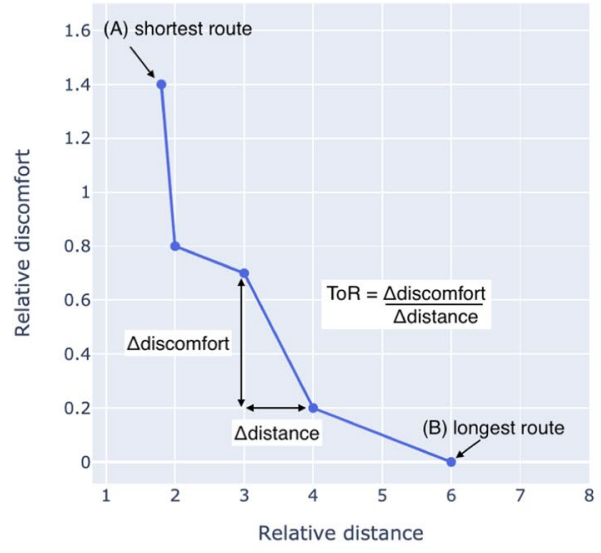

(a)

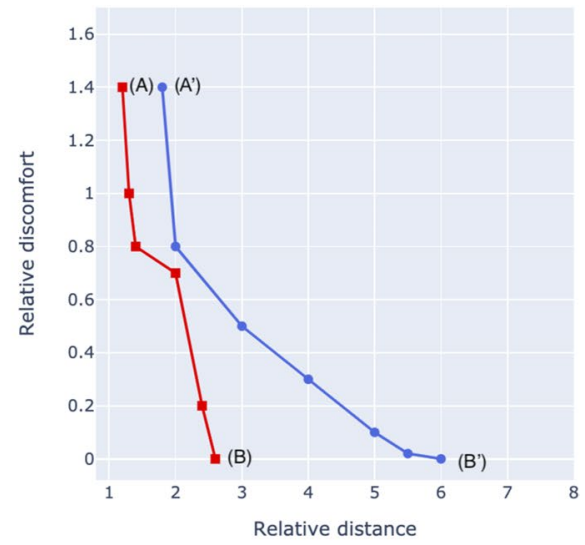

(b)

Fig. 6 Interpretation of bikeability curves. a Extreme routes and trade-off rate are shown. b Provides an example of comparison between two bikeability curves. (Color figure online)

Note that the curves defining Pareto optimal paths can be described by both convex and non-convex functions, where the convexity of the Pareto front depends on the convexity of the feasible set of routes (Brisset and Gillon 2015). As an instance, in Fig. 6b the blue curve is convex whereas the red is non-convex. If we place stronger assumptions on the utility function such as linearity, instead of assumptions 2, some Pareto optima would never be chosen from the users. We prefer not to make strict assumptions on user utility and keep the Pareto front more general.

The ideal bikeability curve, between an OD pair, should resemble a vertical line that reaches the lowest possible discomfort value and with a relative distance close to one. This would guarantee alternative routes that are almost equivalent from the distance aspect, but that offer different discomfort values, including low discomfort values. Moreover, the more routes there are on the bikeability curve, the better because it means that there are many optimally bikeable alternatives. In Fig. $6 \mathrm{~b}$ the red curve is considered as a better bikeability curve compared to the blue curve because the red curve reaches zero discomfort values with a much smaller distance increase, and has more alternative routes.

The result of the methodology is a curve that, combined with cyclists' route preference, shows what bicycle users' the network serves best. To interpret the bikeability curves the analyst should make use of findings on cyclists' actual behaviour and route choice preferences (see Fig. 1 for an overview). This way policymakers are informed on which type of users their network is serving and can define improvement strategies to attract new cyclists.

\section{Network-wide bikeability curve}

There may be a variety of ways to interpret the bikeability curves and use them to evaluate the network bikeability of the whole city. We present an approach that incorporates all the different individual preferences (thus utility functions) and shows how bikeable the network is according to the different users. There are many ways to combine the Pareto fronts of all different OD-pairs into one single so-called network-wide bikeability curve. We will describe an approach that transforms the set of Pareto fronts into one single network-wide bikeability curve. This curve contains for all possible distance-discomfort trade-off choices, the expected 

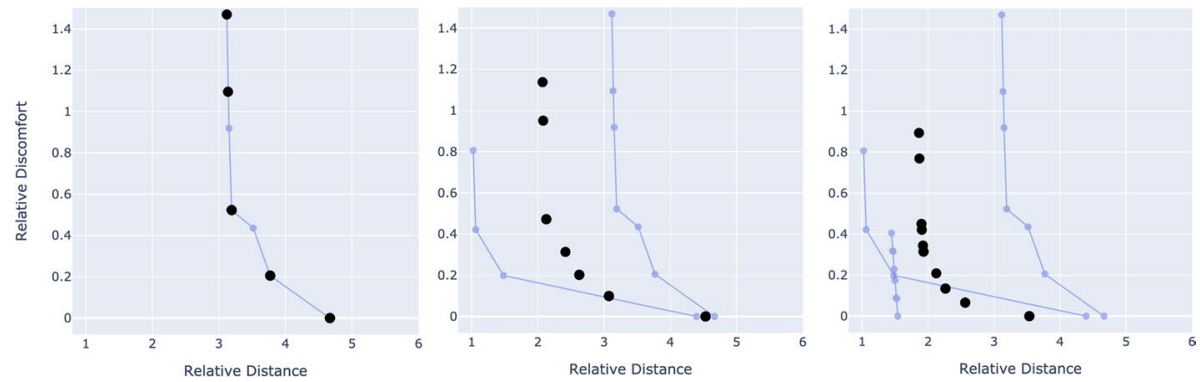

Fig. 7 Building the network-wide Pareto front, for a generic users with utility: $C_{\beta}(d, r)=d+\beta \cdot r$. For this example we computed for values of $\beta=1,2, \ldots, 10$. In reality $\beta$ will be computed for a wider range of values

distance, and discomfort of the optimal route for an OD-pair that is sampled from an ODdemand distribution.

For simplicity, let us assume that people have a linear deterministic cost function: $C_{\beta}(d, r)=d+\beta \cdot r$. Let us now imagine that we have an individual with a certain value of $\beta$. We assign this person to an OD-pair that is sampled from a predefined demand distribution $w$. Precisely, $w(o, d)$ equals the probability that an arbitrary trip in the network has node $o$ as its origin and node $d$ as its destination. We denote $d^{*}$ and $r^{*}$ as the distance and discomfort of this person's optimal trip for the selected OD-pair. Using the demand distribution, we can now express the expected distance $\mathbb{E}\left(d^{*} \mid \beta\right)$ and expected discomfort $\mathbb{E}\left(r^{*} \mid \beta\right)$, given a certain value of $\beta$, as:

$$
\begin{aligned}
& \mathbb{E}\left(d^{*} \mid \beta\right)=\sum_{(o, d) \in N \times N} w_{o, d} \cdot D\left(\pi_{o, d}^{*}(\beta)\right) \\
& \mathbb{E}\left(r^{*} \mid \beta\right)=\sum_{(o, d) \in N \times N} w_{o, d} \cdot R\left(\pi_{o, d}^{*}(\beta)\right),
\end{aligned}
$$

where $D(\cdot)$ and $R(\cdot)$ are the distance and discomfort function respectively and $\pi_{o, d}^{*}(\beta)$ denotes the optimal path from $o$ to $d$ with cost parameter $\beta$, which can be calculated according to Eq. (1):

$$
\pi_{o, d}^{*}(\beta)=\operatorname{argmin}_{\pi \in \Pi_{o, d}} C_{\beta}(D(\pi), R(\pi)),
$$

with $\Pi_{o, d}$ the set of all paths between $o$ and $d$. Similar to the single OD-pair analysis, we notice that it is impossible to express the expected distance and discomfort (i.e. bikeability) as a single point, because of their dependency on the discomfort-distance trade-off parameter $\beta$. In a comparable fashion as for the single OD case, we, therefore, define the networkwide bikeability curve $B$ as the set of the unique expected discomfort versus expected distance points, for all possible $\beta$ :

$$
B=\left\{\left(\mathbb{E}\left(d^{*} \mid \beta\right), \mathbb{E}\left(r^{*} \mid \beta\right)\right) \mid \beta \in \mathbb{R}_{\geq 0}\right\} .
$$

Figure 7 shows how the network-wide bikeability curve builds up starting from one curve and considering one additional curve for each iteration. An interesting property of this network-wide bikeability curve is that it is guaranteed to be convex. This is because we use a linear cost function, which makes that the distance-discomfort point of an optimal 
route is always in the convex hull of a Pareto front. We will not give the details, but it can be shown that the distance-discomfort aggregation [Eqs. (5) and (6)] preserves this property.

\section{Calculation of the network-wide bikeability curve}

The first thing to realize when calculating the network-wide bikeability curve $B$ is that the parameter space $\Pi_{o, d}$ from which to select the optimal path [Eq. (7)] can be reduced to the Pareto front $\Pi_{o, d}^{*}$ for origin $o$ and $d$. To calculate the points in set $B$ [Eq. (8)], we will briefly describe two methods. A simple approach, which is not guaranteed to be $100 \%$ correct, iterates over $\beta$ with a sufficiently small fixed step, such that we expect to not "skip" a point in the curve. Although this simple approach does not guarantee to find all points in $B$, our case-studies indicate that with a small iteration step this approach is capable in revealing the true "shape" of $B$.

If networks become large and/or $100 \%$ coverage is required, a more advanced approach has to be considered. We realize that there is a limited number of values for $\beta$ to be tested in order to be sure that we will find all elements of $B$. The main idea is to first identify the 'critical' values of $\beta$ for each OD-pair, where a 'critical' value is defined as a value for $\beta$ at which the optimal route changes. These 'critical' values can easily be determined in an exact way by matching them with the slopes between consecutive points in the Pareto front. Putting the 'critical' $\beta$ values of all OD-pairs in one large ordered list, we can state that between two consecutive 'critical' $\beta$ values, the result of (7) is guaranteed to be the same in the whole interval between these two values, so it suffices to evaluate only one single point in each interval. This calculation is guaranteed to provide the exact $B$. The choice between the simple and advanced approach boils down to a trade-off between computational time and accuracy. The advanced approach might become slow for very large networks, where the simple approach could still be computed in a reasonable time at the expense of a reduced resemblance of the true $B$.

\section{Implementation of the discomfort function}

Discomfort of a path consists of an edge component, expressed by $r_{e}(\cdot)$, and a node component, expressed by $r_{n}(\cdot)$. Practitioners aiming to implement this methodology can select the discomfort function specifications depending on the analysis they want to conduct. The only condition is that the discomfort has to be positive. Some examples of discomfort function specifications are presented hereafter.

Possible examples of edge discomfort function $r_{e}(\cdot)$ are:

- Distance travelled on the bike-unfriendly network:

$$
r_{e}(e)= \begin{cases}d(e), & \text { if } \mathcal{L}(e) \text { is a bike-unfriendly layer } \\ 0, & \text { otherwise }\end{cases}
$$

- Number of facility changes. One could measure discomfort, or express a component of it, by counting the number of facility changes. 


$$
r_{e}(e)= \begin{cases}1, & \text { if edge } e \text { is an inter-layer link } \\ 0, & \text { otherwise }\end{cases}
$$

Certainly, one could also put specific weights on the different types of facility changes, which demands a deeper empirical insight into the experienced discomfort of all types of maneuvers.

- Accident risk, based on distance and road type. In this case, the discomfort function would be

$$
r_{e}(e)=\alpha_{\ell} \cdot d(e), \quad \ell=\mathcal{L}(e),
$$

where $\alpha_{\ell}$ is a layer-specific constant that defines the accident risk per distance unit on layer $\ell$ and $d(e)$ is the exposure to the risk in terms of distance travelled. The accident risk $\alpha_{\ell}$ will be different depending on the cities, their road regulation, and bike culture. Both the exposure and the probability of an accident can be functions of the volumes of bikes and cars on the streets.

A similar function can also measure discomfort proportionally to the distance travelled on bike infrastructure of different comfort levels. $\alpha_{\ell}$ values will be low (close to or equal to 0 ) for bike-friendly layers and high for bike-unfriendly layers. This discomfort definition is similar to roadway stress used in Lowry and Loh (2017), which is a percentage increase in perceived travel distance.

- The discomfort function can depend on the demand (both at a OD or link-level). As a fact, too many cyclists on the same street segment can slow down the ideal speed of some cyclists and also create queues at intersections that may delay the departure of cyclists at a green light. The challenge to implement such a discomfort function is to obtain real-time estimates of crowdedness on bike streets. Many municipalities in The Netherlands employ inductive loop sensors for traffic signal controllers, through which we can estimate flows or queues in specific locations of the network (Reggiani et al. 2019).

- Bike level of service (BLOS) is an index that attempts to rank bike street segments based on perceived comfort and safety and can be a valid discomfort function for our methodology. For an overview of the methods, we refer to Zuniga-Garcia et al. (2018) however a comparison on BLOS methodologies showed diverse scores of BLOS for the same street segment (Lowry et al. 2012). This shows that there is no agreement on how to grade a street level of service for bicycles.

Below is a list of possible examples of node discomfort function $r_{n}(\cdot)$ :

- Presence of a signalized intersections could be modeled as an impedance factor:

$$
r_{n}(n)= \begin{cases}1, & \text { if node } n \text { is a signalized intersection } \\ 0, & \text { otherwise }\end{cases}
$$

- Expected waiting time at intersections can be a realistic impedance factor of traversing a node. 


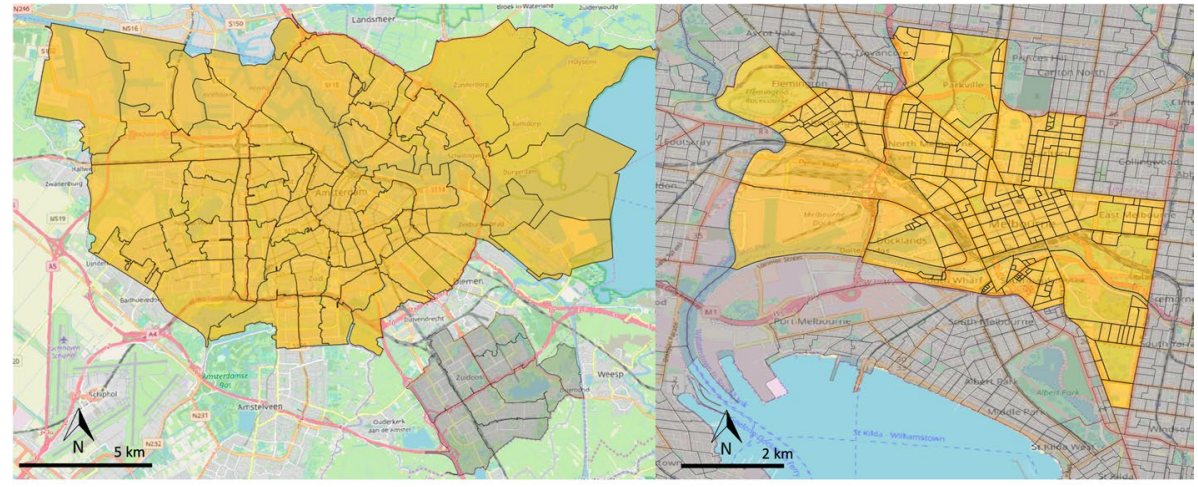

(a) Amsterdam

(b) Melbourne City

Fig. 8 City areas in analysis, the scale for each city is provided at the bottom-left of each map. a Amsterdam, b Melbourne City

\section{Case study: Amsterdam-Melbourne}

In this section, we give an example of how the proposed methodology can be implemented on real street networks and analyse bikeability results between two cities, Amsterdam and Melbourne, with very different bike cultures. We explain the data used for the case study in "Areas of study and data sets" section. The bicycle networks are modeled and coarsegrained in "Modelling the multi-layer networks" section. The results from the bikeability analysis at city and neighbourhood level are presented in "City level bikeability assessment" and "Neighbourhood level bikeability assessment" sections respectively.

\section{Areas of study and data sets}

Melbourne and Amsterdam are selected to show an application of the methodology. These two cities have very different bike cultures, the share of bike trips in Amsterdam and Melbourne is respectively 27\% and 2\% (Pucher and Buehler 2007), which allows us to test if the methodology is able to explain the difference in bike culture from a bikeability point of view. For the network of the city of Melbourne, we consider "Melbourne city" area given the higher densities and consequently the higher bike-share compared to other parts of the city. Concerning the network of the city of Amsterdam, we remove from the network the "Amsterdam-Zuidoost" area since it is a disconnected component of the network. The resulting areas of the two cities are shown in Fig. 8. The size of the two areas is significantly different however, this does not affect the comparison since route distances, in the analysis, are re-scaled according to the shortest distance available.

The case study uses the bike and car street networks made available from Open Street Map project (OSM). Such crowd-sourced and open access platforms provide an attractive and often up-to-date source of detailed data (Ferster et al. 2019). Although we are aware of tagging inconsistency, Ferster et al. (2019) report that OSM can be more updated than municipality records, given the higher frequency with which "the crowd" contributes to updating the OSM compared to the city releasing updated data. Moreover, we are aware of inaccuracies of OSM data such as missing links or disconnected components that in reality are connected. In order to not have bikeability results affected by these inaccuracies, 
we aggregate the urban bicycle network data (from OSM) to city zone level (described in "Modelling the multi-layer networks" section). Namely, two locations could be disconnected due to missing links in OSM, but if their respective zones have uninterrupted paths connecting them we assume that also the specific locations are connected. The benefit of using OSM data is evident for reproducibility reasons as well as ease of accessibility.

The travel demand between zones in Amsterdam has been computed based on the outcome of the learning-based transportation oriented simulation system (ALBATROSS) for the base year 2004 (Arentze and Timmermans 2004). A detailed description of how the modified ALBATROSS data set (calibrated to match Amsterdam's mode split) was derived is available at Winter and Narayan (2019). After filtering out all trips that started or ended outside the city, intra-zone trips and trips with a distance larger than $10 \mathrm{~km},{ }^{4}$ the resulting passenger trips' data set included a total of 52,656 agents performing a total of 139,223 trips.

\section{Modelling the multi-layer networks}

We import the networks from Open Street Map (OSM) using OSMNx package available for python (Boeing 2017). This data is available and easy to retrieve for many cities worldwide, which makes this analysis replicable in other cities as well. We will work with a coarse-grained city network (Hamedmoghadam et al. 2019), rather than the fine-grained urban bicycle network for two reasons: (1) to reduce the computational burden of working with large-scale networks, (2) to be less dependent on OSM inaccuracies, due to crowdsourced nature of the dataset.

Hereafter we explain the meaning of the nodes, links, and layers of the bike network. Given a graph representation of the urban bicycle network $G(N, E, L)$, we build a coarsegrained network $C\left(N^{\prime}, E^{\prime}, L\right)$ such that the structure and weights of the coarse network represent the structural characteristics of the urban bicycle network graph. The network is built with a granularity level of zones (statistical areas in Melbourne and postal code areas in Amsterdam). The set of layers remains unchanged when building the coarse-grained network.

\section{Nodes}

The coarse-grained network $C\left(N^{\prime}, E^{\prime}, L\right)$ has one node per zone per layer, corresponding to the centroid of the zone. This means that a node $n^{\prime} \in N^{\prime}$ corresponds to a set of nodes in $N$. To make this mapping explicit, we introduce $\mathcal{N}: N^{\prime} \rightarrow \mathcal{P}(N)$ as the function that maps a node $n^{\prime} \in N^{\prime}$ in the coarse-grained network on the set of corresponding nodes in the original network.

\section{Links}

For each origin $o^{\prime} \in N^{\prime}$ and destination $d^{\prime} \in N^{\prime}$ in the coarse-grained network, and a layer $\ell \in L$, we define an intra-layer edge $e^{\prime}=\left(\left(o^{\prime}\right)^{\ell},\left(d^{\prime}\right)^{\ell}\right)$ if there exist an origin node

\footnotetext{
${ }^{4}$ We filter based on trip distance and not mode so to do an analysis not on the observed bicycle trips, but on the potential ones. $95 \%$ of cycling trips in the Netherlands are within $10 \mathrm{~km}$, thus the exclusion of longer tips from this study (Schneider 2021).
} 


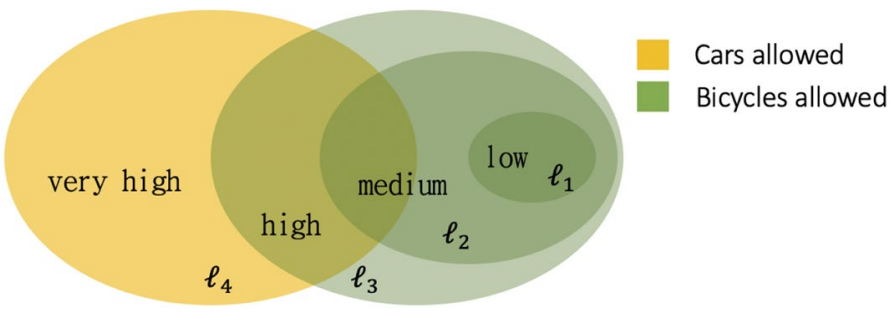

Fig. 9 Diagram of the four layers and their related level of discomfort. To explain the difference between layers, the queries to retrieve these networks are reported in Appendix 1

$o \in \mathcal{N}\left(o^{\prime}\right)$ and destination node $d \in \mathcal{N}\left(d^{\prime}\right)$ in the original network that can be connected by a path that only uses nodes in zones $o^{\prime}$ and $d^{\prime}$ and edges on layer $\ell$.

Each intra-layer edge (i.e. connecting two nodes from the same layer), has a layer-specific length and discomfort associated with the different network-layers. Inter-layer links exist only between the same nodes, and have zero weight associated to them. Thus a user can switch between layers with no discomfort cost via inter-layer links connecting the same nodes on different layers.

\section{Layers}

We import four network layers, corresponding to different OSM facility types. In particular, the four layers corresponds to the following facility type:

1. bike lanes and bike tracks, denoted as $\ell_{1}$,

2. bike lanes, bike tracks and residential streets, denoted as $\ell_{2}$,

3. all streets where cycling is allowed by city laws, denoted as $\ell_{3}$,

4. car streets, denoted as $\ell_{4}$.

We assume that the four layers have increasing levels of discomfort [low, medium, high, very-high] although some elements of one layer may be also included in another layer, as shown in the Venn diagram in Fig. 9. This assumption is made for the sake of simplicity in this case study but can be released (if detailed data on comfort value per network edge is available) without loss of generality. The low discomfort links are contained in the medium discomfort layer and the medium discomfort layers are contained in the high discomfort layer (as green sets show in Fig. 9), because if one tolerates medium discomfort, they can also tolerate lower discomfort links. The car layer includes both very high levels of discomfort as well as some streets with high and medium discomfort.

\section{Edge weights}

Each intra-layer edge $e^{\prime}$ on a particular layer $\mathcal{L}\left(e^{\prime}\right)$ of the coarse-grained network has a length that corresponds to the average shortest distance, on that layer, between all nodes of the zones it is connecting. The average shortest distance is computed as the mean of all shortest paths between the two zones, without routing through any other zone as in the following formula 

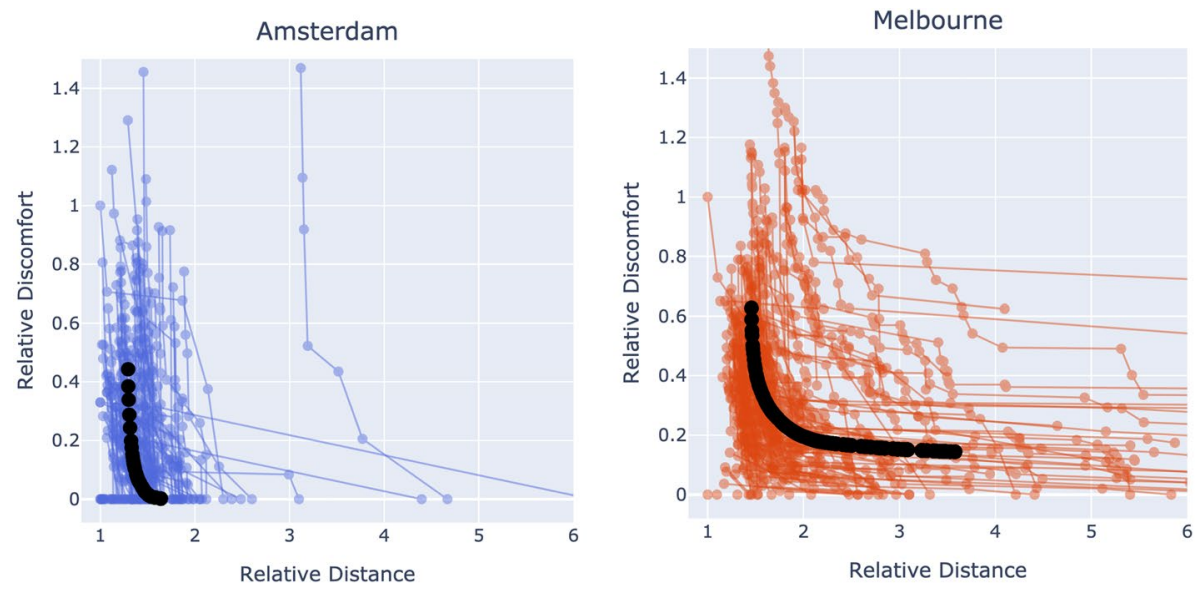

Fig. 10 The red and blue curves are a subset of all individual bikeability curves for each city (one-hundred randomly selected OD pairs). The black points represent the network-wide bikeability curve computed over all OD pairs. (Color figure online)

$$
d\left(e^{\prime}\right)=\frac{\sum_{o \in \mathcal{N}\left(e_{1}^{\prime}\right)} \sum_{d \in \mathcal{N}\left(e_{2}^{\prime}\right)} \Delta\left(o, d, \mathcal{L}\left(e^{\prime}\right)\right)}{\sum_{o \in \mathcal{N}\left(e_{1}^{\prime}\right)} \sum_{d \in \mathcal{N}\left(e_{2}^{\prime}\right)} \delta_{o, d, \mathcal{L}\left(e^{\prime}\right)}},
$$

where $e_{1}^{\prime}$ and $e_{2}^{\prime}$ denote the source and target node of edge $e^{\prime}$, respectively. Reachability $\delta_{o, d, \ell}$ indicates whether or not node $d$ can be reached from node $o$ in the original network $G$, by using only intra-layer links in layer $\ell$ of neighbouring zones. It returns 1 if this is the case, 0 otherwise. $\Delta(o, d, \ell)$ represents the length of the shortest path between $o$ and $d$, using only links in layer $\ell$ of neighbouring zones. By the definition of $e^{\prime}$, this value is always larger than 0 , since $e^{\prime}$ only exists if there is at least one direct path connecting $o^{\prime}$ and $d^{\prime}$ on layer $\mathcal{L}\left(e^{\prime}\right)$.

Each intra-layer edge $e^{\prime}$ on layer $\ell^{\prime}$ has discomfort value corresponding to a predefined discomfort function. As reported in "Implementation of the discomfort function" section many discomfort functions can be defined as long as the value is always nonnegative, in order to satisfy Dijkstra's algorithm requirements. For the sake of simplicity and interpretability of results, in this case study, we define the discomfort function as a percentage of the distance travelled on a specific layer (Lowry and Loh 2017). In particular, we assume equally distanced sequence of values, between zero and one, for the percentage of distance to be considered as discomfort. The higher the discomfort on the layer, the higher the percentage of distance that is considered in the discomfort value, as reported hereafter:

$$
r_{e}\left(e^{\prime}\right)= \begin{cases}d\left(e^{\prime}\right), & \text { if } \mathcal{L}\left(e^{\prime}\right)=\ell_{4} \\ 0.66 \cdot d\left(e^{\prime}\right), & \text { if } \mathcal{L}\left(e^{\prime}\right)=\ell_{3} \\ 0.33 \cdot d\left(e^{\prime}\right), & \text { if } \mathcal{L}\left(e^{\prime}\right)=\ell_{2} \\ 0, & \text { if } \mathcal{L}\left(e^{\prime}\right)=\ell_{1} .\end{cases}
$$

Node discomfort attributes $r_{n}\left(e^{\prime}\right)$ are set to zero since it has no relevance to model node attributes for such a coarse-grained network. 


\section{City level bikeability assessment}

Figure 10 shows the individual and network-wide bikeability curves for the two studied cities. The individual curves from the two cities perform quite differently, confirming the idea that in bike-friendly environments, like Amsterdam, the bicycle network provides more direct and comfortable routes. Almost all OD pairs in Amsterdam have a route alternative with zero discomfort, whereas in Melbourne not all ODs have a zero discomfort option and if they do it may require a very large detour. This means that no matter how much detour a cyclist is willing to take in Melbourne it will not always lead to a discomfort value close to zero. Overall, Amsterdam's curves are more vertically shaped, whereas Melbourne curves are more L-shaped, implying that in Amsterdam there is a larger discomfort decrease per distance increase than in Melbourne.

In addition to the individual bikeability curves Fig. 10 shows the network-wide bikeability curve (in black) as presented in "Network-wide bikeability curve" section. In this part, we assume uniform distribution over all selected ODs (note that this assumption may penalize some cities by considering low performing ODs although they have no bicycle demand between them). Note that strictly speaking the relative distance used by the bikeability curves is the route length divided the Euclidean distance, whereas most of the results in the literature (as in "Literature review" section) use the detour which is route length divided the shortest available route. In order to compare these values, we multiply the detour values by the average street circuity of the city, so to compare the observed route circuity with detour values retrieved from the literature. The average street circuity of Amsterdam is 1.06 and it is 1.05 in Melbourne. ${ }^{5}$

The average bikeability curve of Amsterdam shows that the average trip requires a circuity value between 1.28 and 1.63, from highest to lowest discomfort. This is in line with findings from the literature presented in "Literature review" section, where detour varies between 8 and 93\% (which multiplied by the street circuity of Amsterdam's network results in a route circuity of 8 and 98\%). Amsterdam's average relative distances, for all discomfort levels, reflect detour preferences of both commuters and leisure type preference, thus it provides a network for a wide range of cyclists types, serving both high and low comfort seekers. Discomfort levels on average reach low (close to zero values) on trips with a circuity of 40-60\%, given the overview in "Literature review" section these are acceptable detour values for low traffic stress seekers and off-street bike facilities. Amsterdam's network-wide bikeability curve has lower values of circuity and discomfort compared to Melbourne.

Melbourne's network-wide bikeability curve, instead, includes trips with circuity up to three and a half times longer and does not reach discomfort values below 0.1 . Evidence from revealed and stated preference studies, suggests that detour factors beyond 0.93 (which multiplied by the street circuity of Melbourne's network results in a route circuity of $98 \%$ ) are not commonly accepted by cyclists (especially not by commuters). Hence, the trips most likely chosen by commuter cyclists in Melbourne are on the left part of the curve; with circuity below 0.9 and discomfort above 0.2 . However, those trips do not serve low discomfort acceptance cyclists, since the left part of the curve does not reach zero values (meaning that routes entirely on bike dedicated facilities are not common). The right part of the curve in Melbourne has trips with a large increase of distance (beyond what

\footnotetext{
5 The average street circuity was computed with OSMNX as the average ratio between an edge length and the straight-line distance between the two nodes it links.
} 


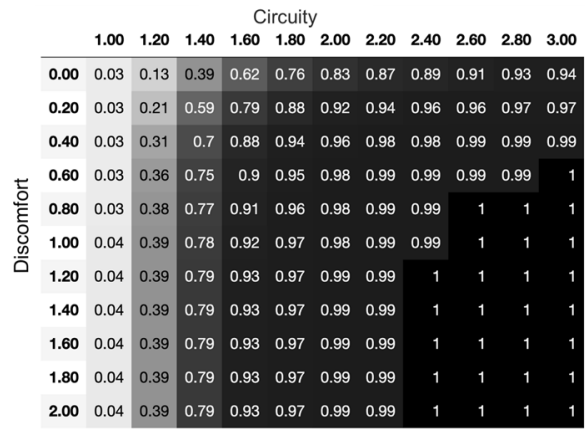

(a) Amsterdam

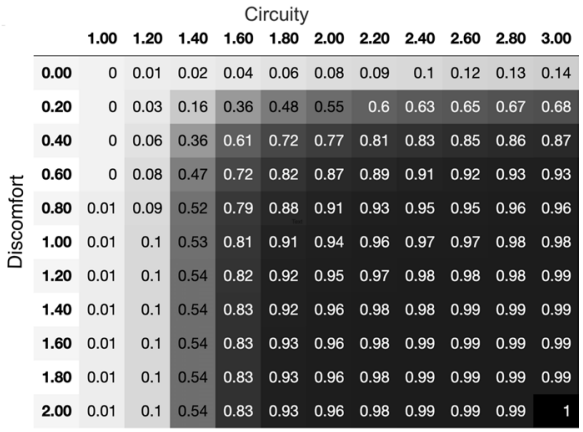

(b) Melbourne

Fig. 11 Network Bikeability tables. Each cell shows the percentage of OD pairs (over all the possible OD pairs) that offer at least one route with discomfort and circuity both lower or equal to the value reported on the column and row header respectively

is acceptable according to the literature (see "Literature review" section) and only minor reduction of discomfort. These route alternatives, on the right side of the curve, do not meet the needs of sporty cyclists or commuters (high discomfort and low detour acceptance) nor of weekend or afternoon cyclists that seek to cycle on off-street facilities (equivalent to discomfort value of zero) and detour smaller than 93\% (Krizek et al. 2007).

\section{Bikeability tables}

In order to have a more systematic approach to interpret how bikeable the network of a city is we build the bikeability table as in Fig. 11. In each cell we report the percentage of OD pairs (over all ODs in the network) that offer at least one route with discomfort and circuity both lower or equal to the value reported on the column and row header respectively. The bikeability tables (in Fig. 11), show that the two cities offer a different ratio of routes with zero discomfort value, across all circuity values (first row of the tables). Zero discomfort value (as defined in the "edge weights" "Modelling the multi-layer networks" section) means that the entire trip is on bike tracks and bike lanes streets (resulting in no increase in perceived travel distance). For example, in Amsterdam 83\% of OD pairs are connected with maximum circuity of 2 . In Melbourne, instead, only $8 \%$ of all the OD pairs are connected with zero discomfort and maximum circuity of 2 . By considering the ratio of routes within a circuity of 1.20 we observe that Amsterdam has $13 \%$ of ODs with zero discomfort, $21 \%$ of ODs with discomfort below or equal to 0.20 , and $31 \%$ of ODs with discomfort below or equal to 0.40 whereas Melbourne respectively has 1\%,3\%, 6\% of ODs. The analysis makes clear that a higher percentage of Amsterdam's routes has a low discomfort value, compared to Melbourne's, given the same circuity factor. Meaning that on average Melbourne serves only low comfort seekers whereas Amsterdam serves both low and high comfort seekers, within acceptable detour rates.

\section{Inclusion of travel demand}

This section analyses how the bikeability curve changes when travel demand on the network is considered. Namely, how well does the network accommodate observed and 
Fig. 12 Comparison between average bikability curve of Amsterdam, with and without demand
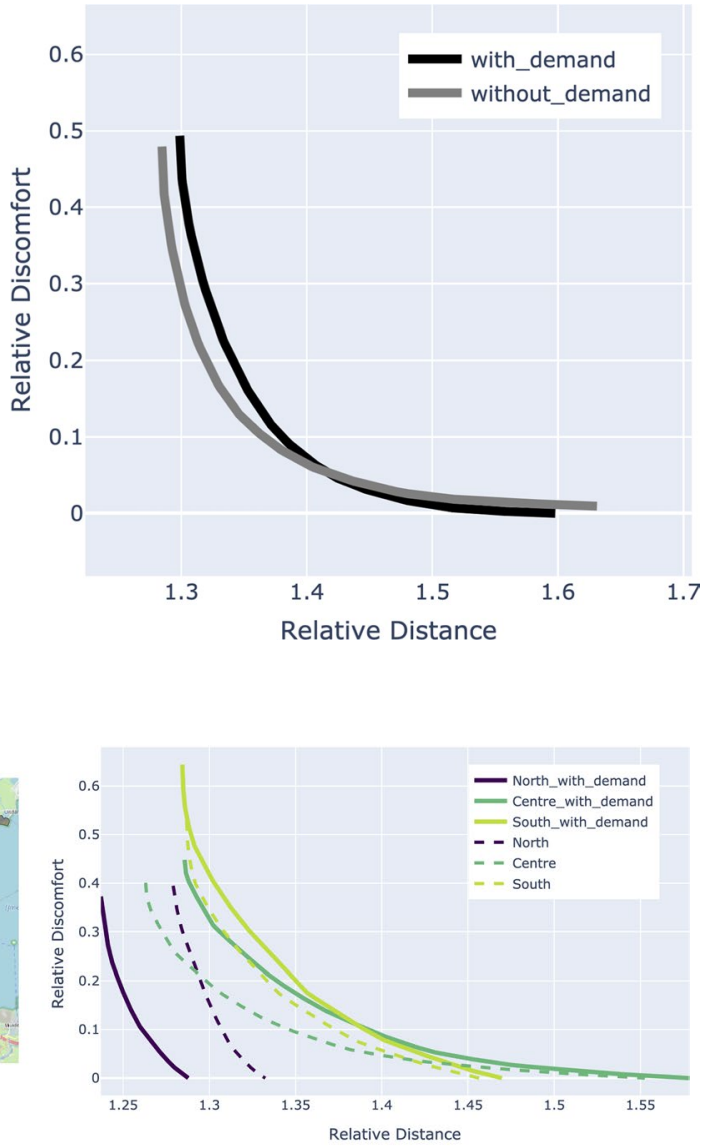

(b)

Fig. 13 Comparison between average bikability curve of north, centre, and south neighbourhood of Amsterdam, with and without demand. a Neighbourhoods under analysis. b Neighbourhood-wide bikeability curves

potential bicycle demand? Increasing connectedness per se can be a waste of resources if there is no demand between two specific zones.

The demand is used to define probability values to weigh each OD pairs (zones) in the network. Including the demand shows a less bikeable average bikeability curve of the whole city. In Fig. 12 the curve weighted on travel demand between zones has higher discomfort values for detour until 1.4, meaning that there is more demand between zones of a city that are not as comfortable nor as direct. There is space for improvement on how the network accommodates demand, especially with regards to the users that do not want to detour more than $40 \%$. Instead, the demand-weighted curve performs better for larger detours values. The overall differences between the analysis with and without demand, at city level scale, are minor. A similar analysis was conducted on a smaller portion of the network, to identify differences within the same city. 


\section{Neighbourhood level bikeability assessment}

In this section we showcase how the methodology can be applied to areas within the same city. This allows to compare areas with the same road standards (e.g. cycling rules and culture). The analysis focuses on north, centre, and south of Amsterdam, as delimited in Fig. 13a.

Analysing the zones without demand (dashed line in Fig. 13b). The centre is serving confident and sporty cyclists better, than the vulnerable or commuters that don't accept high discomfort. In order to cycle with low discomfort in the centre, higher levels of circuity are needed, which makes the low discomfort routes in the centre more suitable for recreational cyclists than commuters, according to findings "Literature review" section. The north part of the city has more direct routes for lower discomfort levels, thus this part of the city serves another type of user compared to the city centre: those who like separated facilities and low detour. Finally, the southern sub-network performs worse, in terms of bikeability, than the centre and north on average. However, for circuity values greater than 1.40 it offers lower discomfort and lower detour rates compared to the centre.

Once the demand is used to weigh bikeability curves (solid lines in Fig. 13b) the average curve of the north improves, meaning that the travel demand is concentrated between zones that have better (more direct and comfortable) routes. The centre and south, instead, perform worse when the curves are weighted with the demand. This shows that the ODs with high concentration of trips, in the centre and south, are not the most bikeable ones. Speculating on the reasons for the better performance of the north compared to the south, when demand is added, we think it is more the infrastructure type (thus comfort level) rather than the circuity to make the difference. If we observe the infrastructure type of the OD pairs with the highest demand we see that the North has more kilometers of medium discomfort $\left(\ell_{2}\right)$ infrastructure and less of high discomfort $\left(\ell_{3}\right)$ infrastructure, compared to the south which has less medium and more high discomfort infrastructure. The inclusion of travel demand in the analysis has shown to have an important effect on the results of the analysis and thus for decision making, since the outcome of the analysis shows substantial differences between the zones.

\section{Discussion}

This section discusses possible applications for practice as well as implications of the proposed methodology for future research. Finally, we acknowledge the limitations related to our methodology.

The presented methodology enables cities to make trade-off analysis for bike network infrastructure. For example, a city may want to improve bikeability among popular origins and destinations. By examining the bikeability curves associated with different OD pairs city planners can pinpoint the locations that need to be better connected for cycling. Then, the improvements can be made by reducing detour on routs with low discomfort or by reducing the discomfort on routes with a low detour. Besides using the proposed methodology to assess the bikeability of existing routes, urban planners can also use it to evaluate and prioritize investments. For example by prioritizing projects that improve the bikiability of the worse performing ODs (according to the OD bikeability curves). 
The proposed methodology to assess the bikeability of an OD pair can be employed by bicycle route planner apps. There are two main limitations in this regard: (1) computational time is far from real-time route guidance and (2) the user should know its tradeoff profile. The first challenge is being investigated by Hrncir et al. (2015, 2017). For the second point, since it is not realistic for cyclists to know their willingness to detour and accepted discomfort quantitatively, one option is to include a stated preference survey to users of the app to quantitatively measure their trade-off profile and recommend them specific routes from all those on the Pareto front.

Users might choose non-optimal routes, ones that lie at a band-with from the indifference curve with maximum utility. Studies in the transport field have developed the idea of indifference curves into indifference bands (Vreeswijk et al. 2013). Future research could generalize the bikeability curves to bikeablity bands by investigating to what extent cyclists are aware of routes on the Pareto front.

We identify two limitations, which do not reducing the validity of the methodology but may represent challenges in the implementation phase. First, the availability of data on discomfort and safety on a network level is not easily accessible. In our case study, we made use of OSM facility types as a proxy for discomfort. However, the discomfort and safety of bicycle facilities can be highly context-dependent. For example, these attributes are influenced by the presence of on-street parking, the volume, and the speed of vehicular traffic, as well as local traffic laws. Secondly, the computational burden of working with large-scale detailed networks, is a concern for network-wide implementation. At this point, high-level analysis on coarse-grained networks is more feasible than on fine-grained ones.

\section{Conclusions}

This work introduced a new methodology to assess bikeability of urban networks free from user preference assumptions, so as to provide an exhaustive overview of what routes the network supplies to its users and not the average cyclist. Bikeability is visualised and analysed through the concept of network-wide "bikeability curve" elaborated in this work. The methodology has shown to provide means to study the relationship between directness and comfort of routes over an entire network by modelling the heterogeneity of streets that build up a bicycle network and not making a priori assumptions on users' preferences between contrasting objectives.

Via a case study, we analysed and compared different bike networks based on largescale real-world topological data, and showed the validity of the proposed methodology. Amsterdam's bicycle network showed to supply users with more direct and comfortable routes compared to Melbourne which on average supplied trips with a significantly longer detour for low levels of discomfort. An application to neighbourhoods within Amsterdam showed that different parts of the city accommodate different types of cyclists, the centre is more for recreational and high detour acceptance cyclists, whereas the northern part has more direct routes, preferred by commuter type of cyclists. The inclusion of travel demand in the analysis has shown to have an important effect on the results of the analysis and is crucial for decision-making for network improvements.

The methodology presented in this paper can be of interest to transport planners and policymakers to understand and evaluate urban bicycle networks without making assumptions on user preferences. Planners will gain extensive insight into the bicycle networks by identifying which type of users (high discomfort acceptance or high detour acceptance 
users) are better accommodated by the network and which origin-destination pairs need to be prioritised for an infrastructural improvement. The generalisability of the methodology allows practitioners to apply it in different scenarios, depending on their data and network evaluation criteria.

\section{Appendix 1: Network queries on OpenStreeMap}

Hereby we report the queries used to extract the bicycle network layers $\left(\ell_{1}, \ell_{2}, \ell_{3}, \ell_{4}\right)$ in "Case study: Amsterdam-Melbourne" section, using the python library OSMnx (imported as "ox"). To install and use the OSMnx package we refer to the decriptive paper from the author of the package (Boeing 2017).

$\ell_{1}$ is defined as:

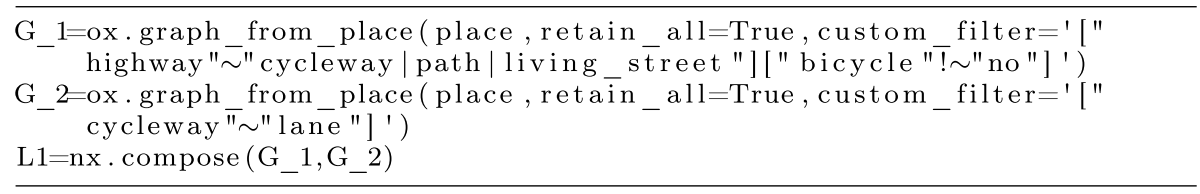

$\ell_{2}$ is defined as:

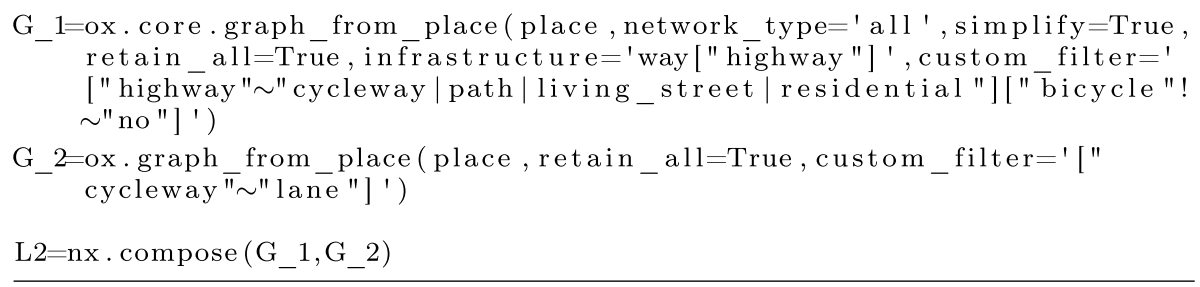

$\ell_{3}$ is defined as:
L3=ox.graph_from_place (place, retain_all=True, network_type='bike', simplif $\bar{y}=$ True $)$

$\ell_{4}$ is defined as:

$\mathrm{L} 4=$ ox .graph_from_place (place, retain_all=True, network_type='drive'
simplify=True)

Acknowledgements This research was supported by the ALLEGRO project, which is financed by the European Research Council (Grant Agreement No. 669792) and the Amsterdam Institute for Advanced 
Metropolitan Solutions. G.R. thanks Prof. Vu for hosting her at Monash University during a visit that initiated this study. GR was supported by the Strategic Alliance Student Exchange Program (SASXP) from the Department of Civil Engineering, Monash University, Melbourne Australia. We are grateful to Ph.D. Konstanze Winter for making available the travel demand data. Finally, we thank the anonymous reviewers for their insightful comments which improved the quality of this work.

Author contributions GR: Conceptualization, methodology, formal analysis, writing-original draft, project administration. TvO: Methodology, mathematical formulation, writing-original draft. HH: Network data processing, writing-review and editing. WD: Conceptualization, supervision, writing-review and editing. LHV: Supervision, writing-review and editing. SH: Conceptualization, supervision, writing-review and editing, funding acquisition.

Code availability A working example of the code is available at: https://github.com/giuliaregg/Understand ingBikability.

\section{Declarations}

Conflict of interest On behalf of all authors, the corresponding author states that there is no conflict of interest.

Open Access This article is licensed under a Creative Commons Attribution 4.0 International License, which permits use, sharing, adaptation, distribution and reproduction in any medium or format, as long as you give appropriate credit to the original author(s) and the source, provide a link to the Creative Commons licence, and indicate if changes were made. The images or other third party material in this article are included in the article's Creative Commons licence, unless indicated otherwise in a credit line to the material. If material is not included in the article's Creative Commons licence and your intended use is not permitted by statutory regulation or exceeds the permitted use, you will need to obtain permission directly from the copyright holder. To view a copy of this licence, visit http://creativecommons.org/licenses/by/4.0/.

\section{References}

Abad, L., van der Meer, L.: Quantifying bicycle network connectivity in Lisbon. Information 9, 287 (2018)

Arellana, J., Saltarín, M., Larrañaga, A.M., González, V.I., Henao, C.A.: Developing an urban bikeability index for different types of cyclists as a tool to prioritise bicycle infrastructure investments. Transp. Res. Part A Policy Pract. 139(January 2019), 310-334 (2020). https://doi.org/10.1016/j.tra.2020.07. 010

Arentze, T.A., Timmermans, H.J.: A learning-based transportation oriented simulation system. Transp. Res. Part B Methodol. 38(7), 613-633 (2004). https://doi.org/10.1016/j.trb.2002.10.001

Ben-Akiva, M.E., Ramming, M.S., Bekhor, S.: Route choice models. In: Schreckenberg, M., Selten, R. (eds.) Human Behaviour and Traffic Networks, pp. 23-45. Springer, Heidelberg (2004)

Boccaletti, S., Bianconi, G., Criado, R., del Genio, C.I., Gómez-Gardeñes, J., Romance, M., Sendiña-Nadal, I., Wang, Z., Zanin, M.: The structure and dynamics of multilayer networks. Phys. Rep. 544(1), 1-122 (2014). https://doi.org/10.1016/j.physrep.2014.07.001. arXiv: 1407.0742

Boeing, G.: OSMnx: new methods for acquiring, constructing, analyzing, and visualizing complex street networks. Comput. Environ. Urban Syst. 65, 126-139 (2017). https://doi.org/10.1016/j.compenvurb sys.2017.05.004

Boisjoly, G., Lachapelle, U., El-Geneidy, A.: Bicycle network performance: assessing the directness of bicycle facilities through connectivity measures, a Montreal, Canada case study. Int. J. Sustain. Transp. 14(8), 620-634 (2020). https://doi.org/10.1080/15568318.2019.1595791

Brisset, S., Gillon, F.: Approaches for Multi-objective Optimization in the Ecodesign of Electric Systems. Elsevier, Amsterdam (2015). https://doi.org/10.1016/B978-1-78242-010-1.00004-5

Broach, J., Dill, J., Gliebe, J.: Where do cyclists ride? A route choice model developed with revealed preference GPS data. Transp. Res. Part A Policy Pract. 46(10), 1730-1740 (2012). https://doi.org/10.1016/j. tra.2012.07.005

Dial, R.B.: A model and algorithm for multicriteria route-mode choice. Transp. Res. Part B 138, 311-316 (1979) 
Dill, J.: Measuring network connectivity for bicycling and walking. 83rd Annual Meeting of the Transportation ... (1):20 (2004). http://reconnectingamerica.org/assets/Uploads/TRB2004-001550.pdf

Duque, D., Lozano, L., Medaglia, A.: An exact method for the biobjective shortest path problem for largescale road networks. Eur. J. Oper. Res (2014). https://doi.org/10.1016/j.ejor.2014.11.003

Ehrgott, M., Wang, J.Y., Raith, A., Van Houtte, C.: A bi-objective cyclist route choice model. Transp. Res. Part A Policy Pract. 46(4), 652-663 (2012). https://doi.org/10.1016/j.tra.2011.11.015

Ferster, C., Fischer, J., Manaugh, K., Nelson, T., Winters, M.: Using OpenStreetMap to inventory bicycle infrastructure: a comparison with open data from cities. Int. J. Sustain. Transp. (2019). https://doi.org/ 10.1080/15568318.2018.1519746

Furth, P.G., Mekuria, M.C., Nixon, H.: Network connectivity for low-stress bicycling. Transp. Res. Rec. J. Transp. Res. Board 2587, 41-49 (2016). https://doi.org/10.3141/2587-06

Garroppo, R.G., Giordano, S., Tavanti, L.: A survey on multi-constrained optimal path computation: exact and approximate algorithms. Comput. Netw. 54, 3081-3107 (2010)

Ghariblou, S., Salehi, M., Magnani, M., Jalili, M.: Shortest paths in multiplex networks. Sci. Rep. 7(1), 1-8 (2017). https://doi.org/10.1038/s41598-017-01655-x

Gholamialm, A., Matisziw, T.C.: Modeling bikeability of urban systems. Geogr. Anal. (2018). https:// doi.org/10.1111/gean.12159

Hamedmoghadam, H., Ramezani, M., Saberi, M.: Revealing latent characteristics of mobility networks with coarse-graining. Sci. Rep. 9(1), 1-10 (2019)

Hansen, P.: Bicriterion path problems. In: Fandel, G., Gal, T. (eds.) Multiple Criteria Decision Making Theory and Application, pp. 109-127. Springer, Heidelberg (1980)

Hood, J., Sall, E., Charlton, B.: A GPS-based bicycle route choice model for San Francisco, California. Transp. Lett. 3(1), 63-75 (2011). https://doi.org/10.3328/TL.2011.03.01.63-75

Hrncir, J., Zilecky, P., Song, Q., Jakob, M.: Speedups for multi-criteria urban bicycle routing. OpenAccess Ser. Inform. 48, 16-28 (2015). https://doi.org/10.4230/OASIcs.ATMOS.2015.16

Hrncir, J., Zilecky, P., Song, Q., Jakob, M.: Practical multicriteria urban bicycle routing. IEEE Trans. Intell. Transp. Syst. 18(3), 493-504 (2017). https://doi.org/10.1109/TITS.2016.2577047

Hyodo, T., Suzuki, N., Takahashi, K.: Modeling of bicycle route and destination choice behavior for bicycle road network plan. Transp. Res. Rec. 1705, 70-76 (2000). https://doi.org/10.3141/1705-11

Jakob, W., Blume, C.: Pareto optimization or cascaded weighted sum: a comparison of concepts. Algorithms 7(1), 166-185 (2014). https://doi.org/10.3390/a7010166

Kang, L., Fricker, J.D.: Bicycle-route choice model incorporating distance and perceived risk. J. Urban Plan. Dev. (2018). https://doi.org/10.1061/(ASCE)UP.1943-5444.0000485

Kellstedt, D.K., Spengler, J.O., Foster, M., Lee, C., Maddock, J.E.: A scoping review of bikeability assessment methods. J. Community Health 46(1), 211-224 (2021). https://doi.org/10.1007/ s10900-020-00846-4

Khorram, E., Khaledian, K., Khaledyan, M.: A numerical method for constructing the Pareto front of multi-objective optimization problems. J. Comput. Appl. Math. 261, 158-171 (2014). https://doi. org/10.1016/j.cam.2013.11.007

Kivelä, M., Arenas, A., Barthelemy, M., Gleeson, J.P., Moreno, Y., Porter, M.A.: Multilayer networks. J. Complex Netw. 2(3), 203-271 (2014). https://doi.org/10.1093/comnet/cnu016. (arXiv1309.7233)

Krizek, K.J., El-Geneidy, A., Thompson, K.: A detailed analysis of how an urban trail system affects cyclists' travel. Transportation 34(5), 611-624 (2007). https://doi.org/10.1007/s11116-007-9130-z

Larsen, J., El-Geneidy, A.: A travel behavior analysis of urban cycling facilities in Montréal, Canada. Transp. Res. Part D Transp. Environ. 16(2), 172-177 (2011). https://doi.org/10.1016/j.trd.2010.07. 011

Lowry, M., Loh, T.H.: Quantifying bicycle network connectivity. Prev. Med. 95, S134-S140 (2017). https://doi.org/10.1016/j.ypmed.2016.12.007

Lowry, M.B., Callister, D., Gresham, M., Moore, B.: Assessment of communitywide bikeability with bicycle level of service. Transp. Res. Rec. 2314, 41-48 (2012). https://doi.org/10.3141/2314-06

Martins, E.Q.V.: On a multicriteria shortest path problem. Eur. J. Oper. Res. 16(2), 236-245 (1984). https://doi.org/10.1016/0377-2217(84)90077-8

Mekuria, M.C., Furth, P.G., Nixon, H.: Low-stress bicycling and network connectivity. Mineta Transp. Inst. Rep. 11-19, 68 (2012)

Menghini, G., Carrasco, N., Schüssler, N., Axhausen, K.W.: Route choice of cyclists in Zurich. Transp. Res. Part A Policy Pract. 44(9), 754-765 (2010). https://doi.org/10.1016/j.tra.2010.07.008

Niaki, M.S., Saunier, N., Miranda-Moreno, L.F.: Analysing cyclist behaviour at cycling facility discontinuities using video data. Trans. Transp. Sci. 9(1), 3-17 (2018). https://doi.org/10.5507/tots.2018.006

Orozco, L.G.N., Battiston, F., Iñiguez, G., Szell, M.: Data-driven strategies for optimal bicycle network growth. Royal Society Open Science (2020) arXiv:1907.07080 
Pucher, J., Buehler, R.: Making cycling irresistible: lessons from the Netherlands, Denmark, and Germany. Transp. Rev. 28(4), 495-528 (2007). https://doi.org/10.1080/01441640701806612

Raith, A.: Speed-up of labelling algorithms for biobjective shortest path problems. In: Proceedings of the 45th Annual Conference of the ORSNZ, pp. 313-322 (2010)

Reggiani, G., Dabiri, A., Daamen, W., Hoogendoorn, S.: Clustering-based methodology for estimating bicycle accumulation levels on signalized links: a case study from the Netherlands. In: 2019 IEEE Intelligent Transportation Systems Conference (ITSC), pp. 1788-1793 (2019)

Schneider, F.: Spatial Activity-Travel Patterns of Cyclists. PhD thesis (2021). https://doi.org/10.4233/ uuid:d72c3cb8-ed98-452b-b8b0-1270556e6367

Schoner, J.E., Levinson, D.M.: The missing link: bicycle infrastructure networks and ridership in 74 US cities. Transportation 41(6), 1187-1204 (2014). https://doi.org/10.1007/s11116-014-9538-1

Sener, I.N., Eluru, N., Bhat, C.R.: An analysis of bicycle route choice preferences in Texas. US. Transp. 36(5), 511-539 (2009). https://doi.org/10.1007/s11116-009-9201-4

Stinson, M.A., Bhat, C.R.: Analysis using a stated preference survey. Transp. Res. Rec. J. Transp. Res. Board 182803, 107-115 (2003)

Ton, D., Cats, O., Duives, D., Hoogendoorn, S.: How do people cycle in Amsterdam, Netherlands? Estimating cyclists' route choice determinants with GPS data from an urban area. Transp. Res. Rec. J. Transp. Res. Board 2662, 75-82 (2017)

Vreeswijk, J.D., Bie, J., van Berkum, E.C., van Arem, B.: Effective traffic management based on bounded rationality and indifference bands. IET Intell. Transp. Syst. 7, 265-274 (2013). https://doi.org/10.1049/ iet-its.2011.0188

Wang, J.Y., Dirks, K.N., Ehrgott, M., Pearce, J., Cheung, A.K.: Supporting healthy route choice for commuter cyclists: the trade-off between travel time and pollutant dose. Oper. Res. Health Care 19, 156164 (2018). https://doi.org/10.1016/j.orhc.2018.04.001

Winter, K., Narayan, J.J.: Amsterdam scenario matsim. (2019). https://doi.org/10.4121/uuid:6108ed857b24-455e-bd95-89d84e6306fa, https://data.4tu.nl/articles/dataset/Amsterdam_Scenario_MATSim/ $12697067 / 1$

Winters, M., Teschke, K., Grant, M., Setton, E.M., Brauer, M.: How far out of the way will we travel? Built environment influences on route selection for bicycle and car travel. Transp. Res. Record 2190(1), 1-10 (2010). https://doi.org/10.3141/2190-01

Zimmermann, M., Mai, T., Frejinger, E.: Bike route choice modeling using GPS data without choice sets of paths. Transp. Res. Part C Emerg. Technol. 75, 183-196 (2017). https://doi.org/10.1016/j.trc.2016.12. 009

Zuniga-Garcia, N., Ross, H.W., Machemehl, R.B.: Multimodal level of service methodologies: evaluation of the multimodal performance of arterial corridors. Transp. Res. Rec. 2672(15), 142-154 (2018). https:// doi.org/10.1177/0361198118776112

Publisher's Note Springer Nature remains neutral with regard to jurisdictional claims in published maps and institutional affiliations.

Giulia Reggiani is a PhD candidate in the ALLEGRO research project at the department of Transport and Planning, Delft University of Technology. She completed BSc and MSc degrees in Management Engineering at Sapienza University of Rome focusing on "Quantitative methods for supporting decisions". Her research interest is on active modes, optimization and machine learning. Her current work focuses on structural properties of bicycle networks worldwide and on data-driven methods for estimating bicycle level of service.

Tim van Oijen is a researcher and programmer at Delft University of Technology. He develops systems to assist in collection, processing and sharing of mobility related data. He also works on crowd monitoring systems that aim to support crowd managers to get better understanding of the crowds behaviour. Apart from his IT-role, Tim van Oijen conducts research on novel route choice and destination estimation techniques, focusing on large-scale event applications.

Homayoun Hamedmoghadam received his M.Sc. degree in Computer Science from Sharif University of Technology, Tehran, Iran, in 2015. He is now with Faculty of Engineering at Monash University, Melbourne, Australia, as a Ph.D. candidate and with School of Engineering at RMIT University, Melbourne, Australia, as a Research Assistant. His research interests include network science, percolation theory, and transportation network analysis. 
Winnie Daamen is currently an Associate professor in the Department of Transport and Planning, Delft University of Technology, The Netherlands. Her research interests include theory, modeling, and simulation of active mode traffic (pedestrians, cyclists). The core of her expertise is in data collection, data analysis to get insight into traffic participant behavior and modeling these behaviors.

Hai L. Vu is a Professor of Intelligent Transport System (ITS) and Director of the Monash Institute of Transport Studies in the Faculty of Engineering, Monash University, Australia. He was a recipient of the 2012 Australian Research Council (ARC) Future Fellowship as well as the Victoria Fellowship Award for his research and leadership in ITS. Prof Vu is a leading expert with 20-years experience who has authored or coauthored over 180 scientific journals and conference papers in the data and transportation network modelling, V2X communications and connected autonomous vehicles (CAVs). His research interests include modelling, performance analysis and design of complex networks, stochastic optimization and control with applications to connected autonomous vehicles, network planning and mobility management.

Serge Hoogendoorn was appointed Antonie van Leeuwenhoek professor Traffic Operations and Management in 2006. He has been the chair of the department Transport and Planning since 2018. He is currently (one of the five) Distinguished Professor of Smart Urban Mobility at Delft University of Technology. Hoogendoorn is the PI Mobility in the Institute of Advanced Metropolitan Solutions (http://www.ams-amste rdam.com). His current research evolves around Smart Urban Mobility, with focal areas such as i) theory, modelling, and simulation of traffic and transportation networks, including cars, pedestrian, cyclists and novel public transport services; ii) development of methods for integrated management of these networks; iii) impact of uncertainty of travel behaviour and network operations; iv) impact of ICT on network flow operations, robustness and resilience, and v) urban data and its applications. In all these topics, his work considers both recurrent and nonrecurrent situations. 